\title{
Escherichia coli em cavalos do Norte do Tocantins e sua relevância na saúde pública
}

\author{
Escherichia coli in Northern Tocantins horses and its relevance in public health \\ Escherichia coli en caballos del Norte de Tocantins y su relevancia en salud pública
}

Recebido: 21/05/2021 | Revisado: 29/05/2021 | Aceito: 03/06/2021 | Publicado: 18/06/2021

Isaura Maria Nunes Madeira

ORCID: https://orcid.org/0000-0002-5760-1638

Prefeitura de Tucumã, Brasil

E-mail: isadoelly@hotmail.com

Karina Almeida Maciel

ORCID: https://orcid.org/0000-0003-4549-1133

Universidade Federal do Tocantins, Brasil

E-mail: maciel.karina@mail.uft.edu.br

Silvia Minharro Barbosa

ORCID: https://orcid.org/0000-0001-5844-5326

Universidade Federal do Tocantins, Brasil

E-mail: minharro@mail.uft.edu.br

Patrícia Duarte Carneiro

ORCID: https://orcid.org/0000-0002-6454-9372

Laboratório de Saúde Pública de Araguaína, Brasil

E-mail:p.duartecarneiro@ hotmail.com

Katyane de Sousa Almeida

ORCID: https://orcid.org/0000-0003-4914-3961

Universidade Federal do Tocantins, Brasil

E-mail: katyanesalmeida@mail.uft.edu.br

Marco Augusto Giannoccaro da Silva

ORCID: https://orcid.org/0000-0001-5844-5326

Universidade Federal do Tocantins, Brasil

E-mail: marcogiannoccaro@mail.uft.edu.br

\begin{abstract}
Resumo
É notório que os equinos são potenciais reservatórios de bactérias zoonóticas, tais como a E. coli e Samonella, porém há pouca informação sobre seu papel como fonte de infecção e sobre o perfil de resistência destes agentes a antibióticos. Neste sentido, objetivou-se verificar a prevalência de E. coli diarreiogênica em equinos do Norte do Tocantins e a susceptibilidade do microrganismo aos principais antimicrobianos empregados em equinos e seres humanos. Amostras de fezes de 72 equinos hígidos foram coletadas da ampola retal e realizou-se a identificação da $E$. coli por meio de provas bioquímicas. A deteç̧ão dos sorogrupos de E. coli diarreiogênica ocorreu empregando-se a técnica de aglutinação em lâmina. A susceptibilidade aos antimicrobianos seguiu a metodologia recomendada pelo CLSI (2011) e NCCLS (2015). Detectou-se E. coli em 39 dos animais estudados e identificou-se 68 diferentes cepas, sendo 32 diarreiogênicas. Destas, 26 eram enteropatogênicas, 5 enteroinvasoras e 1 enterohemorrágica, refletindo o papel dos equinos como reservatórios de estirpes patogênicas e como fonte de infecção a outros animais e seres humanos, representando importante ameaça à saúde pública. A maior sensibilidade e resistência in vitro detectada foi para Ciprofloxacina/Meropenem e Penicilina G, respectivamente. Isolados multirresistentes foram identificados, predominando a resistência concomitante aos $\beta$-lactâmicos, tetraciclinas e sulfonamidas. Conclui-se que os equinos são potenciais reservatórios de E. coli patogênica, que o antibiograma é uma ferramenta indispensável para a escolha do agente antimicrobiano nos casos de colibacilose e, que pessoas que tem contato com equinos devem se atentar à possível transmissão desse patógeno durante o manejo.
\end{abstract}

Palavras-chave: AMR; Antibiograma; Cavalo; Isolamento bacteriano; Zoonose.

\begin{abstract}
It is known that horses are potential reservoirs of zoonotic bacteria, such as Escherichia coli and Salmonella sp. However, there is little information on zoonotic bacteria as potential sources of infection and antibiotic resistance. Thus, the present study aimed to verify the prevalence of diarrheagenic E. coli (DEC) in horses from the northern region of Tocantins, Brazil and determine the microorganism's susceptibility to the main antimicrobials used in horses and humans. By transrectal palpation, stool samples were taken from 72 horses health, and E. coli was identified by biochemical tests. To detect the main DEC serogroups, the slide agglutination technique was performed. The susceptibility to antimicrobials followed the methodology recommended by the Clinical and Laboratory Standards Institute (2011) and the National Committee for Clinical Laboratory Standards (2015). E. coli was detected in 39 of the studied animals and 68 different strains were identified, 32 (47.06\%) of which were DEC. Of these, $26(81.25 \%)$
\end{abstract}


were enteropathogenic, $5(15.62 \%)$ enteroinvasive, and 1 (3.13\%) enterohemorrhagic, which reflects the role of equines as reservoirs of pathogenic strains and as source infection to other animals and humans, representing an important threat to public health. Regarding antimicrobial resistance, the highest in vitro sensitivity and resistance was for Ciprofloxacin/Meropenem and Penicillin G, respectively. Multidrug-resistant isolates were also identified, with predominant resistance to B-lactams, tetracyclines and sulfonamides. It is concluded that equines are potential reservoirs of pathogenic E. coli. An antibiogram is an important tool for the choice of an effective antimicrobial in cases the colibacillosis. People who have contact with equines should pay attention to the possible transmission of these pathogens during management.

Keywords: AMR; Antibiogram; Horse; Bacterial isolation; Zoonosis.

\section{Resumen}

Es bien sabido que los caballos son reservorios potenciales de bacterias zoonóticas, como E. coli y Salmonella, pero hay poca información sobre su papel como fuente de infección y sobre el perfil de resistencia de estos agentes a los antibióticos. En este sentido, el objetivo fue verificar la prevalencia de E. coli diarreiogénica en los caballos del norte de Tocantins y la susceptibilidad del microorganismo a los principales antimicrobianos utilizados en los caballos y seres humanos. Se recolectaron muestras de heces de 72 caballos sanos de la ampolla rectal y se identificó E. coli mediante pruebas bioquímicas. La detección de serogrupos de E. coli diarreiogénica se produjo mediante la técnica de aglutinación en lámina. La susceptibilidad a los antimicrobianos siguió la metodología recomendada por CLSI (2011) y NCCLS (2015). Se detectó E. coli en 39 de los animales estudiados y se identificaron 68 cepas diferentes, 32 de las cuales eran diarreiogénicas. De éstas, 26 eran enteropatogénicas, 5 enteroinvasoras y 1 enterohemorrágica, lo que refleja el papel de los caballos como reservorios de cepas patogénicas y como fuente de infección para otros animales y seres humanos, lo que representa una importante amenaza para la salud pública. La mayor sensibilidad y resistencia in vitro detectada fue para ciprofloxacina/meropenem y penicilina $\mathrm{G}$, respectivamente. Se detectaron aislamientos multirresistentes, predominando la resistencia concomitante a betalactámicos, tetraciclinas y sulfonamidas. Se concluye que los caballos son reservorios potenciales de E. coli patogénica, que el antibiograma es una herramienta indispensable para la elección del agente antimicrobiano en casos de colibacilosis y que las personas que tienen contacto con los caballos deben estar atentas a la posible transmisión de este patógeno durante el trato con los mismos.

Palabras clave: AMR; Antibiogram; Caballos; Aislamento bacteriano; Zoonosis.

\section{Introdução}

Escherichia coli é uma bactéria Gram negativa (Gyles \& Fairbrother, 2010; Tenaillon et al., 2010) que juntamente com outras presentes no ambiente, coloniza em horas o trato intestinal de animais recém-nascidos, permanecendo no organismo de maneira comensal. Porém, alguns sorotipos são patogênicos (Lanz, Kuhnert \& Boerling, 2003; Phillips et al., 2004; Gyles \& Fairbrother, 2010; EFSA, 2017) e responsáveis por altos índices de morbidade e mortalidade em humanos e animais (Lanz, Kuhnert \& Boerling, 2003; Gyles \& Fairbrother, 2010), onde se destaca as E. coli diarreiogênicas (DEC) (Nataro \& Kaper, 1998; Kaper, Nataro \& Mobley, 2004).

Embora a E. coli não seja considerada um patógeno primário grave vinculado a quadros diarreicos em equinos, esta pode atuar como oportunista e agravar o quadro inicial apresentado pelo animal (Duijkeren, Van Asten \& Gaastra, 2000) ou ao ser eliminada peles fezes infectar outras espécies, inclusive o homem, colocando-a como um grande problema de saúde pública, principalmente, se a cepa eliminada for resistente ou multirresistente a antibióticos (Vo et al., 2007; Maddox et al., 2015). Possuir um cavalo é um fator de risco para a transferência de enzimas que conferem resistência à maioria dos antibióticos $\beta$-lactâmicos (Jokisalo et al., 2010), colocando em evidência o risco à saúde pública (De Lagarde et al., 2019).

A resistência antimicrobiana é uma importante ameaça para a medicina humana e veterinária (Who, 2014; Porse et al., 2017) e vem sendo evidenciada na clínica de equinos (Jokisalo et al., 2010; Van Spijk, Schmitt \& Schoster, 2017). Contudo, trabalhos envolvendo dados epidemiológicos e moleculares na espécie são escassos (Maddox et al., 2012; De Lagarde et al., 2019).

Frente ao exposto, objetivou-se com o presente trabalho verificar a prevalência de $E$. coli diarreiogênica em equinos da região Norte do Tocantins bem como a susceptibilidade do microrganismo frente aos principais antimicrobianos empregados em equinos e seres humanos. 


\section{Metodologia}

\section{Caracterização da pesquisa, animais e coleta das amostras}

A pesquisa realizada é do tipo quantitativa exploratória com pesquisa de campo e laboratorial (Fontana, 2018; Mazucato, 2018) em que as amostras de fezes de 72 equinos escolhidos aleatoriamente, sendo 54 machos e 18 fêmeas, com idade média de 7,10 $\pm 3,61$ anos, que pertenciam a 11 diferentes propriedades foram colhidas por palpação transretal utilizandose luva descartável apropriada e imobilização parcial em tronco de contenção específico para a espécie (Casasnovas et al., 2014). Os animais incluídos no estudo não apresentavam sintomas de enfermidade entérica, como diarreia e ou cólica, e nem haviam sido submetidos a antibioticoterapia por um período mínimo anterior de 180 dias. Aproximadamente um grama de fezes foi coletado por animal e imediatamente transferido para um tubo do tipo Falcon, identificado e que continha $2 \mathrm{~mL}$ de PBS-1x ( $\mathrm{NaCl}$ 0,015 M, $\mathrm{PO}_{4}$ 0,01 M, pH 7,2) utilizado para diluição da amostra. Os tubos foram transportados antes e após a coleta em caixa isotérmica contendo gelo reciclável. O tempo entre a coleta da amostra e o início do processamento foi de até duas horas.

\section{Isolamento e Identificação bacteriana}

Uma alíquota da amostra foi inoculada em caldo selenito cistina (Himediaß) e incubada a $37^{\circ} \mathrm{C}$ por 24 horas. Após observação de turvação, o material foi semeado em ágar MacConkey (Himedia®) e incubado a $37^{\circ} \mathrm{C}$, por 24 horas. A identificação da E. coli deu-se por provas bioquímicas seguindo a metodologia proposta por Krieg e Holt (1984) e Gomes e Hernandes (2015). As amostras confirmadas como E. coli foram semeadas em tubo contendo ágar nutriente para conservação.

\section{Detecção dos sorogrupos de E. coli por aglutinação em lâmina}

Amostras previamente armazenadas em ágar nutriente foram repicadas em ágar BHI (Brain Heart Infusion) (SigmaAldrich $\left(\right.$ ) e incubadas em estufa a $37^{\circ} \mathrm{C}$ por 24 horas. Os testes foram realizados de acordo com a recomendação do fabricante.

Para determinação dos sorogrupos EHEC foi utilizado o soro anti E. coli O157 (Probac do Brasil®), para os EPEC, o soro polivalente anti E. coli enteropatogênica clássica (Polivalente A, B e C) (Probac do Brasil®) e para os EIEC o soro polivalente anti E. coli enteroinvasora (Polivalente A e B) (Probac do Brasil®). A presença de aglutinação era considerada resposta positiva para avaliação.

\section{Teste de susceptibilidade aos antimicrobianos}

As cepas estocadas em ágar nutriente foram repicadas em ágar BHI e incubadas a $37^{\circ} \mathrm{C}$ por 24 horas, para crescimento e obtenção de colônias isoladas. A susceptibilidade aos antimicrobianos (antibiograma) seguiu a metodologia recomendada pelo CLSI (2011) e NCCLS (2015), o qual é baseado no método de Bauer et al. (1966). Como controle do teste utilizou-se a cepa de referência E. coli ATCC 25922.

Os antibióticos utilizados foram: betalactâmico associado a inibidor de betalactamase (Amoxicilina com Ácido

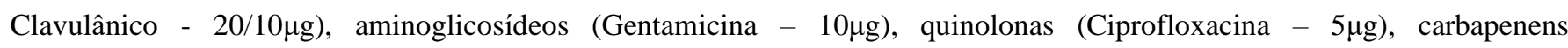

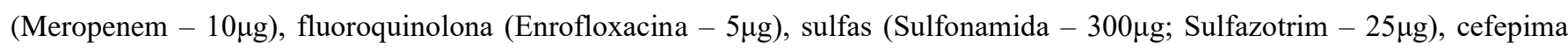
$(30 \mu \mathrm{g})$, florfenicol $(30 \mu \mathrm{g})$ e tetraciclina $(30 \mu \mathrm{g})$. A leitura do teste foi interpretada de acordo com a tabela proposta pelo fabricante (Cefar®) (Laborclin $®)$.

\section{Análise Estatística}

Os dados foram apresentados de forma descritiva e os resultados expressos em suas frequências absolutas e relativas (Sampaio, 2015). 


\section{Resultados e Discussão}

Na presente pesquisa isolou-se E. coli em 39 dos 72 cavalos testados $(54,16 \%)$ e identificou-se por meio da sorologia 68 diferentes cepas, das quais 32 (47,06\%) eram diarreiogênicas (DEC). Destas, 26 (81,25\%) eram enteropatogênicas (EPEC), $05(15,62 \%)$ enteroinvasoras (EIEC) e 01 (3,13\%) enterohemorrágica (EHEC).

Foi descrito que a $E$. coli é facilmente isolada nas fezes de animais com e sem diarreia e que é a principal bactéria que coloniza o intestino dos animais domésticos sadios, sendo o intestino grosso o local de predileção (Tenaillon et al., 2010). Seu papel comensal foi atribuído à absorção de vitaminas e de impedir a fixação de bactérias patogênicas, devido à ocupação da mucosa intestinal (Ferreira \& Knöbl, 2009). Porém, embora exista esta relação de mutualismo, a E. coli possui estirpes patogênicas, como o patótipo diarreiogênico (Croxen \& Finlay, 2010), que podem ser nocivas à saúde humana e animal (Lopes et al., 2016) e, por isso, o isolamento e identificação são importantes, por permitir diferenciar as patogênicas das não patogênicas, realizar investigações epidemiológicas (Gyles \& Fairbrother, 2010) e estabelecer, devido ao convívio próximo do homem com o cavalo, a relação entre infecção animal e a saúde pública.

Assim como nas aves (McPeake, Smyth \& Ball, 2005), a infecção por E. coli nos equinos ocorre principalmente de forma secundária (Duijkeren et al., 2000), no entanto a infecção pode agravar quadros primários e também atingir os seres humanos, uma vez que os equinos atuam como reservatórios de cepas patogênicas e zoonóticas como as EPEC e EHEC, o que demonstra a forte relação com a saúde humana (Vo et al., 2007; Maddox et al., 2015; Souza et al., 2017) e a indissociabilidade entre saúde animal, humana e ambiental.

Embora a maioria das infecções em humanos foram relacionadas ao consumo de alimentos de origem bovina, água contaminada com fezes bovinas ou contato direto com esta espécie, outras fontes de contaminação existem e não foram completamente elucidadas (Beuchat, 2002). Dentre elas, estão o uso de estrume animal inadequadamente tratado (Wilson et al., 2009) para a adubação da terra, principalmente, em hortas residenciais, de agricultura familiar, de baixa produção e na agricultura sustentável. Foi demonstrado durante inoculação experimental, que a E. coli O157:H7 tem um poder de sobrevivência em fezes de equinos muito superior a encontrada em ovelhas, bovinos e porcos (Williams et al., 2008).

No mesmo sentido, em propriedades de baixa produção, o cavalo ainda é costumeiramente empregado como animal de tração e suas fezes eliminadas durante o trabalho, podem contaminar o solo a ser utilizado bem como vegetais a serem consumidos (Lengacher et al., 2010).

Nos animais, a EPEC, é considerada um dos mais importantes patótipos (Gomes \& Hernandes, 2015), pois promove lesão no epitélio intestinal do tipo A/E (attaching and effacing) (Gomes \& Hernandes, 2015; Kaper et al., 2004), desencadeando resposta inflamatória e alterando morfologicamente as microvilosidades (Knutton, Lloyd \& McNeish, 1987), o que resulta clinicamente em diarreia aquosa com muco, febre e desidratação (Rodríguez-Angeles, 2002). Em humanos é a principal responsável por enteroinfecções, tanto em adultos como em crianças (Chandran \& Mazumder, 2013). Contudo, nenhum animal inserido no estudo apresentava alteração compatível com distúrbio gastroentérico (cólica e ou diarreia) e por se tratar de portadores assintomáticos/carreadores assintomáticos que desempenham importante papel como fonte de infecção a outros indivíduos, revela um grande problema à saúde animal e pública. Nos seres humanos, estes portadores assintomáticos podem albergar e disseminar a bactéria por semanas ou meses (Gomes \& Hernandes, 2015; Huijbers et al., 2013; Klemm, Hancock \& Schembri, 2007), fato que pode ocorrer também com os equinos.

Sendo o cavalo um animal domesticado e com contato próximo ao homem, a chance de transmissão de agentes infecciosos aumenta (Hernandes et al., 2009) e aponta à necessidade de inclusão dos equinos nas ações sanitárias de impacto na saúde pública (Rodríguez-Angeles, 2002), fato muitas vezes esquecido. Cepas de E. coli foram isoladas em fezes de criadores e de seus cavalos, indicando uma fonte comum de contaminação ou a transmissão zoonótica entre o cavalo e o criador (Lengacher et al., 2010). Outra relação estabelecida foi no caso de infecção em uma criança que havia visitado três dias 
antes do aparecimento dos sintomas uma pequena fazenda que possuía alguns animais (cabras, um pônei, uma vaca e seu bezerro e dois cães) e isolou-se o mesmo agente (E. coli O157:H7) do pônei e de um dos cães (Trevena et al., 1996). Em um estudo realizado na Coreia, os autores descreveram como potencial fator de risco para a infecção de pessoas ligadas diretamente aos equinos, o contato frequente com estes animais (Chung et al., 2016). Ainda, a contaminação de águas superficiais por animais que albergam a E. coli e a possível contaminação do homem também foi levantada (Chandran \& Mazumder, 2013). Todas as situações expostas sustentam a relação sanidade animal e saúde pública e a necessidade da adoção adequada de medidas sanitárias, implementadas após levantamento epidemiológico nas populações, incluindo-se os equinos neste cenário.

$\mathrm{O}$ isolamento de uma EHEC pode ser destacado como um grande achado deste estudo, uma vez que apenas os bovinos são considerados como os principais reservatórios deste agente (Bardiau et al., 2012; Caprioli et al., 2005; Manning et al., 2008). Muitos bovinos por ela infectados não apresentam sinais clínicos, porém constituem reservatório para outro bovinos, o homem (Gyles, 2007; Gyles \& Fairbrother, 2010) e os equinos (Lengacher et al., 2010). Nosso achado corrobora com esta afirmação, pois quase que a totalidade dos animais estudados $(88,89 \%)$ tinham contato com bovinos e ou pequenos ruminantes. É relevante mais uma vez destacar que os animais desta pesquisa não manifestavam qualquer tipo de sintoma e, assim como os bovinos, desempenham papel de portadores assintomáticos (Caprioli et al., 2005), disseminando silenciosamente o agente no ambiente e infectar o homem. Neste caso, estes podem desenvolver desde diarreia com ou sem colite hemorrágica a quadros mais graves como a síndrome urêmica hemolítica (Bardiau et al., 2012).

Quanto à resistência antimicrobiana, testou-se as amostras dos 39 animais que apresentaram E. coli nas fezes e a maior sensibilidade in vitro detectada foi para os antimicrobianos ciprofloxacina e meropenem, enquanto a maior resistência foi para a penicilina $G$, inexistindo para esta a formação de qualquer halo. Sulfonamida, amoxicilina e doxiciclina também foram os antimicrobianos aos quais as bactérias apresentaram grande resistência. Ainda, em alguns animais identificou-se isolados multirresistentes, ou seja, resistência a pelo menos um antibiótico de três classes diferentes, predominando os betalactâmicos, tetraciclinas e sulfonamidas.

Desde 1970 aponta-se o aparecimento de bactérias resistentes em animais devido ao emprego de antimicrobianos nos animais por médicos veterinários e populares (Mateu \& Martin, 2001) e a transmissão de bactérias multirresistentes entre animais de estimação e seres humanos também já foi descrita (Guardabassi, Schwarz \& Lloyd, 2004; Moyaert et al., 2006; Umber \& Bender, 2009).

Cães e gatos já foram apontados como fontes potenciais para a propagação de resistência antimicrobiana devido ao uso frequente antimicrobianos na rotina veterinária e ao contato muito próximo destes animais com os seres humanos (Guardabassi, Schwarz \& Lloyd, 2004; Moyaert et al., 2006; Umber \& Bender, 2009).

Em equinos, o uso desta classe de medicamento respalda-se na necessidade de tratamento de processos infecciosos diversos e na profilaxia operatória (Robinson \& Sprayberry, 2009), porém o uso racional, correto e baseado no antibiograma é indispensável (Mateu \& Martin, 2001), fato que nem sempre ocorre, principalmente, quando o uso é feito por leigos ou sem prescrição de médico veterinário. Foi demonstrado que cavalos saudáveis possuíam em sua microbiota isolados de E. coli multirresistentes e enzimas inativadoras de $\beta$-lactâmicos (ESBL/AmpC) (Lengacher et al., 2010).

Em estudo retrospectivo foi observado que a penicilina apresentou resistência em 99\% das vezes que foi testada in vitro e que bactérias multirresistentes estavam presentes em 39\% das amostras analisadas em um período de quatro anos (Levandowski, Daroit \& Dos Santos, 2019).

Antibióticos considerados mais antigos como a penicilina e sulfonamida têm apresentado maior resistência em relação aos demais existentes. Desde 1999 (Williams, 1999), a presença das $\beta$-lactamases tem sido reportada como o principal mecanismo de resistência para os $\beta$-lactâmicos, sendo a enzima penicinilase, a responsável pela hidrólise do anel $\beta$-lactâmico e 
a resistência às penicilinas (Mehta et al., 2016).

O uso indiscriminado de antibióticos foi apontado como o principal fator para a seleção e disseminação de bactérias multirresistentes (Laxminarayan et al., 2016), porém a transmissão horizontal de genes resistentes por meio da conjugação mediada por plasmídeos deve ser considerada (Matamoros et al., 2017). A E. coli é uma das bactérias envolvidas nesta transmissão devido à sua ampla distribuição e frequente exposição a antibióticos (Porse et al., 2017).

Os resultados encontrados neste estudo podem estar relacionados ao aumento do uso de antibióticos e seu uso indiscriminado assim como à transferência horizontal de genes de virulência entre os agentes. Cabe destacar que é frequente nas propriedades o uso de antibióticos sem prescrição, assim como o uso de dosagens inadequadas que pode contribuir para a resistência (Mayrhofer et al., 2006). Ainda, observa-se no Norte do Estado do Tocantins, que os antimicrobianos mais empregados em equinos devido ao seu baixo custo são os a base de penicilina, o que corrobora aos achados desta pesquisa quanto à maior resistência.

\section{Conclusão}

Conclui-se com os resultados encontrados que os equinos são potenciais reservatórios de E. coli patogênica e capazes de atuar como fonte de infeç̧ão para os seres humanos e outros animais. Ainda, que testes de sensibilidade in vitro são importantes para a escolha do melhor agente antimicrobiano para que o sucesso terapêutico seja alcançado e um melhor prognóstico para o paciente seja atingido. Por fim, que pessoas que tem contato com os equinos devem se atentar à possível transmissão desses patógenos e manter boas práticas de higiene como a lavagem frequente das mãos e o não consumo de alimentos durante o manuseio dos cavalos em tarefas diversas, além de comunicar o convívio com a espécie ao médico no momento da consulta frente a caso de gastroenterite.

\section{Agradecimentos}

O presente trabalho foi realizado com apoio da Coordenação de Aperfeiçoamento de Pessoal de Nível Superior Brasil (CAPES) - código de financiamento 001 e Programa Nacional de Cooperação Acadêmica na Amazônia PROCAD/Amazônia - CAPES; Laboratório de Microbiologia de Alimentos da Universidade Federal do Tocantins por todo apoio técnico.

\section{Referências}

Bardiau, M., Taminiau, B., DupreZ, J. N., Labrozzo, S. \& Mainil, J. G. (2012). Comparison between a bovine and a human enterohaemorrhagic Escherichia coli strain of serogroup O26 by suppressive subtractive hybridization reveals the presence of atypical factors in EHEC and EPEC strains. FEMS Microbiology Letters. 330(2): 132-139. 10.1111/j.15746968.2012.02542.x

Bauer, A. W., Kirby, W. M. M., Sherris, J. C. \& Turck, M. (1966). Antibiotic susceptibility testing by a standardized single disc method. American Journal of Clinical Pathology. 45(4): 493-496. 10.1093/ajcp/45.4_ts.493

Beuchat, L. R. (2002). Ecological factors influencing survival and growth of human pathogens on raw fruits and vegetables. Microbes and Infection. 4(4): 413423. 10.1016/s1286-4579(02)01555-1

Caprioli, A., Morabito, S., Brugère, H. \& Oswald, E. (2005). Enterohaemorrhagic Escherichia coli: emerging issues on virulence and modes of transmission. Veterinary Research. 36(3): 289-311. 10.1051/vetres:2005002

Casasnovas, A. F., Ayuda, T. C. \& Abenia, J. F. (2014). Manejo e Comportamento. In: Casasnovas A. F., Ayuda T. C. \& Abenia, J. F. (Orgs). A exploração clínica do cavalo. 1ed. São Paulo: Editora MedVet, pp.23.

Chandran, A. \& Mazumder, A. (2013). Prevalence of diarrhea-associated virulence genes and genetic diversity in Escherichia coli isolates from fecal material of various animal hosts. Applied Environmental Microbiology. 79(23): 7371-7380. 10.1128/AEM.02653-13

Chung, Y. S., Song, J. W., Kim, D. H., Shin, S., Park, Y. K., Yang, S. J., Lim, S. K., Park, K. T. \& Park, Y. H. (2016). Isolation and characterization of antimicrobial-resistant Escherichia coli from national horse racetracks and private horse-riding courses in Korea. Journal of Veterinary Science. 17(2): 199206. https://doi.org/10.4142/jvs.2016.17.2.199 
Clinical and Laboratory Standards Institute (CLSI). (2011). Performance standards for Antimicrobial Susceptibility Testing; Twenty-first Informational Supplement. CLSI document M100-S21. 31(1). Wayne, PA: Clinical and Laboratory Standards Institute.

Croxen, M. A. \& Finlay, B. B. (2010). Molecular mechanisms of Escherichia coli pathogenicity. Nature Reviews Microbiology. 8(1): 26-38. $10.1038 /$ nrmicro2265

De Lagarde, M., Larrieu, C., Praud, K., Schouler, C., Doublet, B., Guillaume, S., Fairbrother, J. M. \& Arsenau, T. J. (2019). Prevalence, risk factors, and characterization of multidrug resistant and extended spectrum $\beta$-lactamase/AmpC $\beta$-lactamase producing Escherichia coli in healthy horses in France in 2015. Journal of Veterinary Internal Medicine. 33(2): 902-911. http://10.1111/jvim.15415

Duijkeren, E. V., Van Asten, A. J. A. M. \& Gaastra, W. (2000). Characterization of Escherichia coli isolated from adult horses with and without enteritis. Veterinary Quarterly. 22(3): 162-166. 10.1080/01652176.2000.9695048

European Food Safety Authority (EFSA). (2017). The European Union summary report on antimicrobial resistance in zoonotic and indicator bacteria from humans, animals and food in 2015. EFSA Journal. 15: 212.

Ferreira, A. J. P. \& Knöbl, T. Colibacilose. In: Berchieri Jr., A., Silva, E., Di Fábio, J., Sesti, L. \& Zuanaze, M. A. F. (Orgs). (2009). Doença das aves. 2ed. Campinas: Facta, pp.457-500.

Fontana, F. (2018). Técnicas de pesquisa. In Mazucato, T. (Org.). Metodologia da pesquisa e do trabalho científico (pp. 59-66). Penápolis, São Paulo: FUNEPE.

Gomes, T. A. T. \& Hernandes, R. T. (2015). Escherichia coli Enteropatogênica (EPEC). In: Trabulsi L. R. \& Alterthum F. (Eds). Trabulsi-Alterthum Microbiologia. 6ed. São Paulo: Atheneu, pp.303-10.

Guardabassi, L., Schwarz, S. \& Lloyd, D. H. (2004). Pets animals as reservoirs of antimicrobial-resistant bacteria. Journal of Antimicrobial Chemotherapy. 54(2): 331-332. 10.1093/jac/dkh332

Gyles, C. L. (2007). Shiga toxin-producing Escherichia coli: an overview. Journal of Animal Science. 85(13): 45-62. 10.2527/jas.2006-508

Gyles, C. L. \& Fairbrother, J. M. (2010). Escherichia coli. In: Gyles C.L, Prescott J.F., Songer J.G. \& Thoen C.O. (Eds). Pathogenesis of Bacterial Infections in Animals. 4ed. Iowa: Blackwell Publishing, pp.231-265. 10.1002/9780470958209.ch15

Hernandes, R. T., Elias, W. P., Vieira, M. A. M. \& Gomes, T. A. T. (2009). An overview of atypical enteropathogenic Escherichia coli. FEMS Microbiol Letters. 297(2): 137-49. 10.1111/j.1574-6968.2009.01664.x

Huijbers, P. M. C., De Kraker, M., Graat, E. A. M., Van Hoek, A. H. A. M., Van Santen, M. G., De Jong, M. C. M., Van Duijkeren, E. \& De Greef, S. C. (2013). Prevalence of extended-spectrum ß-lactamase-producing Enterobacteriaceae in humans living in municipalities with high and low broiler density. Clinical Microbiology and Infection. 19(6): 256-59. 10.1111/1469-0691.12150

Jokisalo, J., Bryan, J., Legget, B., Abbott, Y. \& Katz, L. M. (2010). Multiple-drug resistant Acinetobacter baumannii bronchopneumonia in a colt following intensive care treatment. Equine Veterinary Education. 22(6): 281-286. 10.1111/j.2042-3292.2010.00071.x

Kaper, J. B., Nataro, J. D. \& Mobley, H. L. T. (2004). Pathogenic Escherichia coli. Nature Reviews Microbiology. 2(2): 123-140. https://doi.org/10.1038/nrmicro818

Klemm, P., Hancock, V. \& Schembri, M. A. (2007). Mellowing Out: adaptation to commensalism by Escherichia coli asymptomatic bacteriuria strain 83972. Infection and Immunity. 75(8): 3688-3695. http://10.1128/IAI.01730-06

Knutton, S., Lloyd, D. R. \& McNeish, A. S. (1987). Adhesion of enteropathogenic Escherichia coli to human intestinal enterocytes and culture human intestinal mucosa. Infection and Immunity. 55(1): 69-77.

Krieg, N. R. \& Holt, H. J. (1984). Facultatively anaerobic gram-negative rods: Family I. Enterobacteriaceae. In: Krieg N.R. \& Holt H.J. (Eds). Bergey's Manual of Systematic Bacteriology. 1ed. Baltimore: Williams \& Wilkins, pp.408-420.

Lanz, R., Kuhnert, P. \& Boerlin, P. (2003). Antimicrobial resistance and resistance gene determinants in clinical Escherichia coli from different animal species in Switzerland. Veterinary Microbiology. 91(1): 73-84. https://doi.org/10.1016/S0378-1135(02)00263-8

Laxminarayan, R., Sridhar, D., Blaser, M., Wang, M. \& Woolhouse, M. (2016). Achieving global targets for antimicrobial resistance. Science. 353(6302): 874-875. https://doi.org/10.1126/science.aaf9286

Lengacher, B., Kline, T. R., Harpster, L., Williams, M. L. \& Lejeune, J. T. (2010). Low prevalence of Escherichia coli O157:H7 in horses in Ohio, USA. Journal of Food Protection. 73(11): 2089-2092. 10.4315/0362-028X-73.11.2089

Levandowski, R., Daroit, L. \& Dos Santos, L. R. (2019). Escherichia coli: antimicrobial susceptibility monitoring 2015 - 2018 in the north region of the state of Rio Grande do Sul, Southern Brazil. Revista de Ciência Veterinária e Saúde Pública. 6(2): 251-261. 10.4025/revcivet.v6i2.47685

Lopes, E. S., Maciel, W. C., Teixeira, R. S. C., Albuquerque, A. H., Vasconcelos, R. H., Machado, D. N.; Bezerra, W. G. A. \& Santos, I. C. L. (2016). Isolamento de Salmonella spp. e Escherichia coli de psittaciformes: relevância em saúde pública. Arquivo do Instituto Biológico. 83(e0602014): 1-10. 10.1590/1808-1657000602014

Maddox, T. W., Clegg, P. D., Diggle, P. J., Wedley, A. L., Dawson, S., Pinchbeck, G. L. \& Williams N. J. (2012). Cross-sectional study of antimicrobialresistant bacteria in horses. Part 1: Prevalence of antimicrobial-resistant Escherichia coli and methicillin-resistant Staphylococcus aureus. Equine Veterinary Journal. 44(3): 289-296. 10.1111/j.2042-3306.2011.00441.x 
Maddox, T. W., Clegg, P. D., Williams, N. J. \& Pinchbeck, G. L. (2015). Antimicrobial resistance in bacteria from horses: epidemiology of antimicrobial resistance. Equine Veterinary Journal. 47(6): 756-765. https://doi.org/10.1111/evj.12471

Manning, S. D., Motiwala, A. S., Springman, A. C., Qi, W., Lacher, D. W., Ouellette, L. M.; Mladonicky, J. M., Somsel, P., Rudrik, J. T., Dietrich, S. E., Zhang, W., Swaminathan, B., Alland, D. \& Whittam, T.S. (2008). Variation in virulence among clades of Escherichia coli O157:H7 associated with disease outbreaks. Proceedings of the National Academy of Sciences of the United States of America. 105(12): 4868-73. 10.1073/pnas.0710834105

Matamoros, S., Van Hattem, J. M., Arcilla, M. S., Willemse, N., Melles, D. C., Penders, J., Vinh, T. B., Hoa, N. T., Bootsma, M. C. J., Van Genderen, P. J., Goorhuis, A., Grobusch, M., Molhoek, N., Oude Lashof, A. M. L., Stobbering, E. E., Verbrug, H. A., De Jong, M. D. \& Schultsz, C. (2017). Global phylogenetic analysis of Escherichia coli and plasmids carrying the mcr-1 gene indicates bacterial diversity but plasmid restriction. Scientific Reports. 7(15364): 1-9. 10.1038/s41598-017-15539-7

Mateu, E. \& Martin, M. (2001). Why is anti-microbial resistance a veterinary problem as well? Journal of Veterinary Medicine Series B-Infectious Diseases and Veterinary Public Health. 48(8): 569-81. 10.1046/j.1439-0450.2001.00475.x

Mayrhofer, S., Paulsen, P., Smulders, F. J. M. \& Hilbert, F. (2006). Antimicrobial resistance in commensal Escherichia coli isolated from muscle foods as related to the veterinary use of antimicrobial agents in food-producing animals in Austria. Microbial Drug Resistance. 12(4): 278-283. $10.1089 / \mathrm{mdr} .2006 .12 .278$

Mazucato, T. (Org.). (2018). O projeto de pesquisa. In Mazucato, T. (Org.). Metodologia da pesquisa e do trabalho científico (pp. 45-47). FUNEPE.

Mazucato, T. (Org.). (2018). Métodos. In Mazucato, T. (Org.). Metodologia da pesquisa e do trabalho científico (pp. 58). Penápolis, São Paulo: FUNEPE.

McPeake, S. J. W., Smyth, J. A. \& Ball, H. J. (2005). Characterization of avian pathogenic Escherichia coli (APEC) associated with colisepticaemia compared to faecal isolates from healthy birds. Veterinary Microbiology. 110(3-4): 245-253. 10.1016/j.vetmic.2005.08.001

Mehta, S. C., Samanta, M., Chow, D. C. \& Palzkill, T. (2016). Avoiding the Carbapenem trap: KPC-2 $\beta$-lactamase sequence requirements for Carbapenem hydrolysis. The FASEB Journal. 30(S1) Suppl.: 1083.20-1083.20

Moyaert, H., De Graef, E. M., Haesebrouck, F. \& Decostere, A. (2006). Acquired antimicrobial resistance in the intestinal microbiota of diverse cat populations. Research in Veterinary Science. 81(1): 1-7. 10.1016/j.rvsc.2005.10.004

Nataro J. P. \& Kaper J. B. (1998). Diarrheagenic Escherichia coli. Clinical Microbiology Reviews. 11(1): $142-201$.

National Committee for Clinical Laboratory Standards (NCCLS). (2015). Performance standards for antimicrobial disk and dilution susceptibility tests for bacteria isolates from animals. 3ed. CLSI supplement VET01S. Wayne, PA.

Normanno, G., Corrente, M., La Salandra, G., Dambrosio, A., Quaglia, N. C., Parisi, A., Greco, G., Bellacicco, A. L., Virgilio, S. \& Celano, G. V. (2007). Methicillin-resistant Staphylococcus aureus (MRSA) in foods of animal origin product in Italy. International Journal of Food Microbiology. 117(2): 219-22. 10.1016/j.ijfoodmicro.2007.04.006

Phillips, I., Casewell, M., Cox, T., De Groot, B., Friis, C., Jones, R., Nightingale, C., Preston, R. \& Waddell, J. (2004). Does the use of antibiotics in food animals pose a risk to human health? Journal of Antimicrobial Chemotherapy. 53(1): 28- 52. 10.1093/jac/dkg483

Porse, A., Gumpert, H., Kubicek-Sutherland, J. Z., Karami, N., Adlerberth, I., Wold, A. E.; Andersson, D. I., Sommer, M. O. A. (2017). Genome dynamics of Escherichia coli during antibiotic treatment: transfer, loss, and persistence of genetic elements in situ of the infant gut. Frontiers in cellular and infection microbiology. 7:126. 10.3389/fcimb.2017.00126

Robinson, N. E. \& Sprayberry, K. A. (2009). Current therapy in equine medicine. 6ed. St Louis: Elsevier Saunders Co., pp.1104.

Rodríguez-Angeles, G. (2002). Principales características y diagnóstico de los grupos patógenos de Escherichia coli. Salud Pública de México. 44(5): 464-475

Sampaio, I. B. M. (2015). Estatística aplicada à experimentação animal. 4ed. Belo Horizonte: Fundação de Estudo e Pesquisa em Medicina Veterinária e Zootecnia (FEPMVZ), pp.265.

Souza, C. O., Melo, T. R. B., Melo, C. S. B., Menezes, E. M., De Carvalho, A. C. \& Monteiro, L. C. R. (2016). Escherichia coli enteropatogênica: uma categoria diarreiogênica versátil. Revista Pan-Amazônica de Saúde. 7(2): 79-91. 10.5123/S2176-62232016000200010

Tenaillon, O., Skurnik, D., Picard, B. \& Denamur, E. (2010). The population genetics of commensal Escherichia coli. Nature Reviews Microbiology. 8(3): 207-217. 10.1038/nrmicro2298

Trevena, W. B., Hooper, R. S., Wray, C., Willshaw, G. A., Cheasty, T. \& Domingue, G. (1996). Vero cytotoxin-producing Escherichia coli O157 associated with companion animals. Veterinary Record. 138(16): 400.

Umber, J. K. \& Bender, J. B. (2009). Pets and antimicrobial resistance. Veterinary Clinics of North America: Small Animal Practice. 39(2): 279-92. 10.1016/j.cvsm.2008.10.016

Van Spijk, J. N., Schmitt, S. \& Schoster, A. (2017). Infections caused by multidrug-resistant bacteria in an equine hospital (2012-2015). Equine Veterinary Education. 31(12): 653-658. 10.1111/eve.12837

Vo, A. T. T., Van Duijkeren, E., Fluit, A. C. \& Gaastra, W. (2007). Characteristics of extended-spectrum cephalosporin-resistant Escherichia coli and Klebsiella pneumoniae isolates from horses. Veterinary Microbiology. 124(3-4): 248-55. 10.1016/j.vetmic.2007.04.027

Williams, J. D. (1999). $\beta$-lactamases and $\beta$ - lactamase inhibitors. International Journal of Antimicrobial Agents. 12(Suppl 1): 3-7. 10.1016/s09248579(99)00085-0 
Research, Society and Development, v. 10, n. 7, e17310716383, 2021

(CC BY 4.0) | ISSN 2525-3409 | DOI: http://dx.doi.org/10.33448/rsd-v10i7.16383

Williams, A. P., McGregor, K. A.; Killham, K. \& Jones, D. L. (2008). Persistence and metabolic activity of Escherichia coli O157:H7 in farm animal faeces. FEMS Microbiology Letters. 287(2): 168-73. 10.1111/j.1574-6968.2008.01310.x

Wilson, R. S., Parker, J., Kovacs, D., Doohan, D. \& Lejeune, J. (2009). Contamination prevention and response related to fresh and fresh-cut produce: an expert perspective on the farmer decision making process. Food Protection Trends. 29(8): 488-492.

World Health Organization (WHO). (2014). Antimicrobial resistance: global report on surveillance. pp.257. 